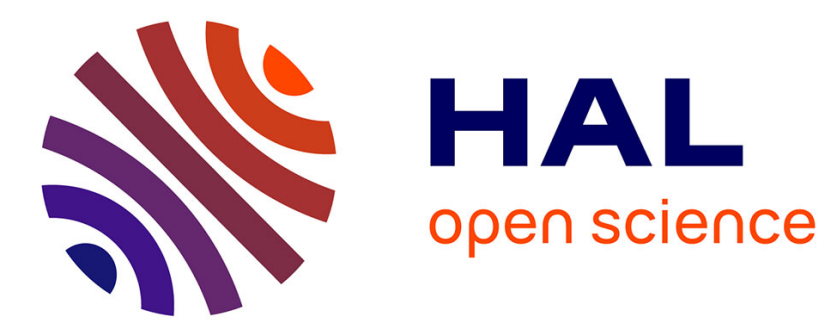

\title{
Probing Conformers of Benzene Dimer with Intermolecular Coulombic Decay Spectroscopy
}

Nicolas Sisourat, Sévan Kazandjian, Tsveta Miteva

\section{To cite this version:}

Nicolas Sisourat, Sévan Kazandjian, Tsveta Miteva. Probing Conformers of Benzene Dimer with Intermolecular Coulombic Decay Spectroscopy. Journal of Physical Chemistry A, 2016, 10.1021/acs.jpca.6b09501 . hal-01427205

\section{HAL Id: hal-01427205 https://hal.sorbonne-universite.fr/hal-01427205}

Submitted on 5 Jan 2017

HAL is a multi-disciplinary open access archive for the deposit and dissemination of scientific research documents, whether they are published or not. The documents may come from teaching and research institutions in France or abroad, or from public or private research centers.
L'archive ouverte pluridisciplinaire HAL, est destinée au dépôt et à la diffusion de documents scientifiques de niveau recherche, publiés ou non, émanant des établissements d'enseignement et de recherche français ou étrangers, des laboratoires publics ou privés. 


\title{
Probing Conformers of Benzene Dimer with
}

\section{Intermolecular Coulombic Decay Spectroscopy}

\author{
Nicolas Sisourat, ${ }^{*}$ Sévan Kazandjian, and Tsveta Miteva \\ Sorbonne Universités, UPMC Univ Paris 06, CNRS, Laboratoire de Chimie Physique \\ Matière et Rayonnement, F-75005, Paris, France \\ E-mail: Nicolas.Sisourat@upmc.fr
}

${ }^{*}$ To whom correspondence should be addressed 


\begin{abstract}
Benzene dimer is a prototype to study intermolecular interactions between aromatic systems. Owing to the weak interactions between the molecules within the dimer, several conformational geometries are nearly isoenergetic and thus coexist even at low temperatures. Furthermore, standard spectroscopies are unable to distinguish between them. In this work, we study the electronic relaxation processes following inner-valence ionization of benzene and the lowest conformers of benzene dimer. We show that the kinetic energy distributions of the secondary electrons emitted via two autoionization mechanisms, namely the Auger and the Intermolecular Coulombic Decay (ICD) effects, provide a means to probe the conformers of benzene dimer. The proposed spectroscopy opens the way to a better characterization of weakly-bound molecular clusters.
\end{abstract}




\section{Introduction}

Aromatic $\pi-\pi$ interactions are ubiquitous in chemistry, biology and engineering: these noncovalent interactions play a vital role in molecular recognition processes, ${ }^{1}$ in the tertiary and quaternary structures of proteins and $\mathrm{DNA}^{2,3}$ as well as in organic crystal growth. ${ }^{4}$

Benzene dimer is the simplest prototype to study intermolecular interactions between aromatic systems. As such it has been intensively investigated for decades $\left(\operatorname{see}^{5}\right.$ for a recent review). Owing to the small binding energy between the two molecules $(\approx 2.8 \mathrm{kcal} / \mathrm{mol}$, i.e. $120 \mathrm{meV}$ ), the dimer may exist in different conformational geometries, even at low temperatures. An accurate description of such large weakly-bound systems is a challenging task for quantum chemists and advanced ab initio methods must be employed to provide reliable energies. ${ }^{6-8}$ Theory predicts three low and nearly isoenergetic conformers, namely the Tshaped (T), the tilted T-shaped (TT) and the parallel-displaced (PD) geometries (see Fig. 1). Among the other conformers lying above these lowest configurations the sandwich (S) geometry has been widely investigated since it represents a model for stacked structures. The two T-shaped conformers have been observed experimentally using microwave spectroscopy. ${ }^{9}$ However, the other conformers are more challenging to identify because they lack permanent dipole moments. Furthermore, photoelectron and photoion spectroscopies, which are powerful analytical tools, cannot resolve the different conformers owing to the small chemical shift between the latter. ${ }^{10-12}$

In this work, we propose a spectroscopic method based on the Intermolecular Coulombic Decay (ICD) effect ${ }^{13-16}$ which can help to identify the different conformers. ICD is an efficient and ultrafast energy transfer process: after inner-valence ionization of a molecule embedded in a chemical environment this molecular ion transfers its excess energy to a neighboring molecule which is then ionized. The energy transfer can take place over huge distances: for example, in the giant helium dimer, ${ }^{17,18}$ it was demonstrated that energy transfer can be achieved via ICD over up to $14 \AA$. Furthermore, the timescale for the energy transfer and the kinetic energy distribution of the secondary electron (so-called ICD electron) 


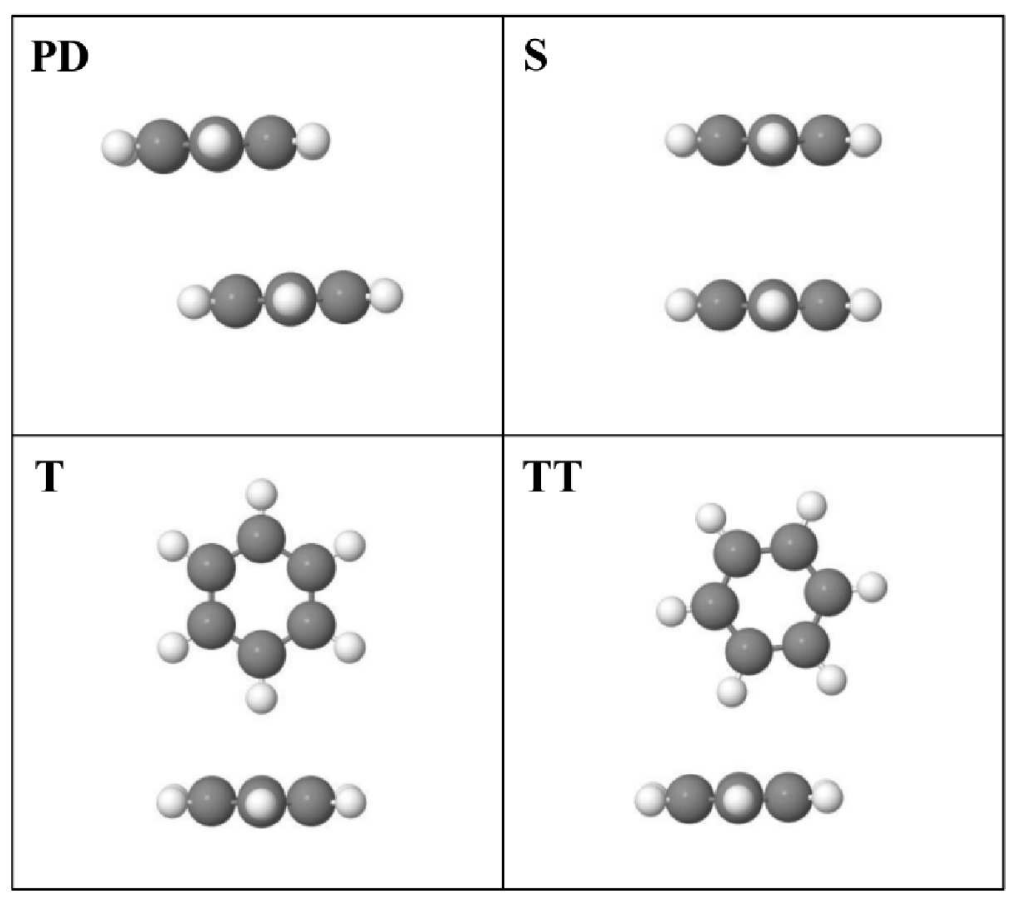

Figure 1: Geometries of the parallel-displaced (PD), sandwich (S), T-shaped (T) and tilted T-shaped (TT) conformers of benzene dimer taken from Ref. ${ }^{22}$

depend strongly on the environment and in particular on the distance between the molecules. Therefore this electronic decay process can be used as a spectroscopic tool for probing the chemical environment of a molecule. For example, ICD has recently been used as a probe for structural analysis ${ }^{19}$ : Fasshauer et al. have shown that with the help of ICD one can retrieve information about the geometric structures of mixed rare-gas clusters.

ICD may be triggered by photoionization from the inner-valence shell of one molecule within the dimer and leads to the emission of a slow ICD electron. For deep inner-valence ionization of benzene molecule intramolecular autoionization processes (i.e. Auger decay) are also allowed: an outer-valence electron from the ionized molecule fills the vacancy and a second outer-valence electron (so-called Auger electron) from that molecule is ejected. We show here that the kinetic energy distribution of the photoelectron reported in coincidence with that of the ICD and Auger electrons may be used to identify the benzene dimer conformers. It should be noted that Auger decay and ICD are expected to take place within less 
than 100 fs which rules out the possibility for molecules in each conformer to rotate before emitting the secondary electron. The electron-electron coincidence spectra reflect therefore the initial geometry of the dimers.

\section{Computational Methods}

The ground state equilibrium geometry of the isolated benzene molecule was obtained by energy minimization at the Moller-Plesset 2nd order (MP2) level using the MOLPRO package. ${ }^{20,21}$ The geometries of the conformers of the benzene dimer considered in this work were taken from Ref. ${ }^{22}$ (see Fig. 1).

The single (IP) and double (DIP) ionization potentials were computed with Algebraic Diagrammatic Construction (ADC) methods ( $\operatorname{see}^{23}$ for a recent review). The single ionization potentials for the five systems were computed using the non-Dyson extended second-order $\mathrm{ADC}(2) \mathrm{x}$ scheme for the one-particle Green's function. ${ }^{24,25}$ Such a method provides accurate spectroscopic amplitudes and binding energies for outer-valence and inner-valence ionized states of fairly large systems. The $\mathrm{ADC}(2)$ method for the two-particle propagator ${ }^{26-28}$ was used to compute the double ionization potentials of all species. $\mathrm{ADC}(2)$ has been successfully used for the study of dense double ionization spectra of molecules and clusters (see ${ }^{27}$ and references therein).

The Hartree-Fock energies, molecular orbitals and the integrals needed for the ADC calculations were computed with a split-valence triple-zeta basis set ${ }^{29}$ using the GAMESSUS package. ${ }^{30}$ Convergence of the results with respect to the basis set was checked.

The kinetic energy distributions of the photoelectrons coming from inner-valence ionization of a monomer reported in coincidence with that of the secondary electrons were estimated with the following model:

- A two-hole population analysis ${ }^{27}$ is used to determine the type of decay. ICD final states are characterized by the presence of one hole on each molecule while after the Auger 
decay the two holes are localized on the same molecule which is initially ionized. ElectronTransfer-Mediated-Decay (ETMD) ${ }^{31,32}$ is another decay process in which one electron from the neighboring benzene molecule is transferred to the ionized monomer and the excess energy is used to eject a second electron. In this case, the two holes in the final states are therefore localized on the molecule which is not initially ionized.

- For each singly-ionized state computed with the ADC method the energy distribution of the secondary electron (if any decay channels are open) is given by the difference between the energy of the singly-ionized states and the energies of the accessible doubly-ionized states.

- We assume that the branching ratio for Auger decay (when this channel is open) is ten times larger than ICD. This approximation is justified because Auger decaytime is usually below 10 fs while ICD takes place within 100 fs. ${ }^{33}$ ETMD is completely neglected since it is efficient only at short intermolecular distances. ${ }^{31}$ Furthermore, for the Auger channels the singlet final states contribute more to the Auger spectra than the triplet ones ${ }^{34,35}$ whereas in ICD the triplet states contribute three times more than the singlet states. ${ }^{36}$ Thus we multiply by 3 the corresponding contributions. Finally, for a given decay process (Auger or ICD) and a given spin symmetry all weights are set equal.

- Denoting the weight for a given channel (decaying state $i$ and final state $j$ ) as $\omega_{i, j}$, the spectroscopic amplitudes as $p_{i}$ and the binding energy and the secondary electron energy as $B E$ and $E$ respectively, the electron-electron concidence spectrum is given by

$$
\sigma(B E, E)=\sum_{i=1}^{N_{I P}} \sum_{j=1}^{N_{D I P}} p_{i} \omega_{i, j} e^{-\alpha\left[\left(B E-B E_{i}\right)^{2}+\left(E-E_{j}\right)^{2}\right]}
$$

where $N_{I P}$ and $N_{D I P}$ are the number of ADC singly- and doubly-ionized states, respectively. We assume that each peak has a Gaussian shape, with a full width at half maximum set to $0.5 \mathrm{eV}$, in order to approximately include vibrational broadening. We use binding energy instead of photoelectron kinetic energy since the former does not depend on the photon energy. 


\section{Results and Discussion}

\section{Isolated benzene molecule}

The kinetic energy distributions of the photoelectron are obtained directly from the single ionization potentials, whereas the kinetic energy distributions of the secondary electron can be estimated from the IP and the DIP (see section Computational Methods). We first discuss the IP and DIP of isolated benzene. The experimental and theoretical valence photoelectron spectra of isolated benzene molecule are known ( $\operatorname{see}^{37,38}$ and references therein). The spectrum of the benzene dication has also been measured and computed (see ${ }^{35,39}$ and references therein). These previous data are used to benchmark our calculations (see Table I). The computed single and double ionization potentials of isolated benzene are shown in Fig. 2. The spectra were shifted such that the lowest line of each spectrum matches the experimental lines: we used $+0.48 \mathrm{eV}$ for the single ionization potentials, -0.20 and +0.40 $\mathrm{eV}$ for the singlet and triplet double ionization potentials, respectively. The relatively small values of these shifts demonstrate the accuracy of the methods used in this work.

Table 1: Lowest ADC single and double ionization potentials (in eV) of isolated benzene molecule compared to experimental values. The ionization potentials shown in Figs. 2-5 are shifted such that these lowest ADC ionization potentials match the experimental ones.

\begin{tabular}{|c|c|c|c|c|c|c|}
\hline & IP $($ ADC $(2) \mathrm{x})$ & IP $\left.^{(\text {Expt. }}\right)^{38}$ & \multicolumn{2}{|c|}{ DIP $(\mathrm{ADC}(2))$} & \multicolumn{2}{|c|}{ DIP (Expt.) } \\
\hline & - & - & Triplet & Singlet & Triplet & Singlet \\
\hline Benzene & 8.77 & 9.25 & 24.25 & 25.45 & 24.65 & 25.25 \\
\hline
\end{tabular}

The ground state equilibrium geometry of benzene is planar with $\mathrm{D}_{6 h}$ symmetry and electronic valence configuration $\left(2 \mathrm{a}_{1 g}\right)^{2}\left(2 \mathrm{e}_{1 u}\right)^{4}\left(2 \mathrm{e}_{2 g}\right)^{4}\left(3 \mathrm{a}_{1 g}\right)^{2}\left(2 \mathrm{~b}_{1 u}\right)^{2}\left(1 \mathrm{~b}_{2 u}\right)^{2}\left(3 \mathrm{e}_{1 u}\right)^{4}\left(1 \mathrm{a}_{2 u}\right)^{2}$ $\left(3 \mathrm{e}_{2 g}\right)^{4}\left(1 \mathrm{e}_{1 g}\right)^{4}$. The lowest single ionization potentials correspond to the ionization of the outer valence electrons. The inner-valence region is located above 18-20 eV where many lines with small spectroscopic amplitudes are seen. This is characteristic of the breakdown of the molecular orbital picture ${ }^{40}$ reflecting strong electronic correlation: in this energy range, 
the singly-ionized states cannot be described as ionization from a single molecular orbital. Instead electronic configurations corresponding to ionization of one electron and excitation of another electron into an unoccupied orbital contribute significantly to each electronic state. However, the fairly intense peaks just below $26 \mathrm{eV}$ may still be approximately associated with the ionization of $\left(2 \mathrm{e}_{1 u}\right)$ orbitals which are mostly built on carbon 2 s atomic orbitals. As shown below, the inner-valence region is around the double ionization threshold of the molecule and is of particular interest for this study.

The lowest DIPs appear at $24.65 \mathrm{eV}$ for the triplet and $25.25 \mathrm{eV}$ for the singlet states. These peaks correspond to double ionization from the highest occupied molecular orbital (HOMO) $\left(1 \mathrm{e}_{1 g}\right)$. Higher energy peaks are associated with ionization of an electron from the HOMO and of successively deeper outer-valence electrons. Few lines are seen at binding energy below $26 \mathrm{eV}$. Higher-lying states are then grouped around $28 \mathrm{eV}$ and then $30 \mathrm{eV}$. Each line in the spectra corresponds to the energy threshold for which the Auger decay channels open.

Using the IP and DIP as well as the model presented in section Computational Methods, we computed the binding energy-Auger electron energy coincidence spectrum, which is shown in Fig 3. The Auger decay channel is energetically closed for the lowest states of the singlyionized molecule. Therefore, the first band in the coincidence spectrum appears only at around $26 \mathrm{eV}$ binding energy. The corresponding Auger electron energy distribution is below $1 \mathrm{eV}$ since the first inner-valence ionized states lie just above the double ionization threshold. For higher binding energies, more Auger channels open which is seen in the broader energy distribution of the secondary electron.

\section{Conformers of benzene dimer}

We now consider the IP of the four conformers of benzene dimer (see lower panels of Fig. 4). Only the inner-valence region is shown. Compared to the spectrum of the isolated molecule, the binding energy spectra are richer for all conformers. Furthermore, the peaks are red- 
Figure 2: Single (lower panel) and double ionization (upper panel) potentials of isolated benzene molecule. Singlet and triplet doubly-ionized states are indicated as black and green lines, respectively.

shifted due to the polarization effect of the neighboring molecule. The most intense peaks corresponding to the ionization from the $\left(2 \mathrm{e}_{1 u}\right)$ orbitals are then located around $25 \mathrm{eV}$. The difference in the spectra between the conformers is rather small such that the most intense peaks of the different conformers strongly overlap. However, it should be noted that for the sandwich geometry numerous states with small spectroscopic amplitudes are grouped together at around $22 \mathrm{eV}$ while for the other geometries the states are more evenly distributed at higher energies. As discussed below, these peaks are characterisic of the sandwich conformer and may be used to spectroscopically distinguish it from the other conformers.

The DIP of the dimers, shown in the upper panels of Fig. 4, are even more different from that of the isolated molecule. In particular, several states appear around $20 \mathrm{eV}$ which correspond to single ionization of both molecules within the dimer and are thus attributed to ICD final states. Furthermore, the double ionization potentials are more sensitive to the 


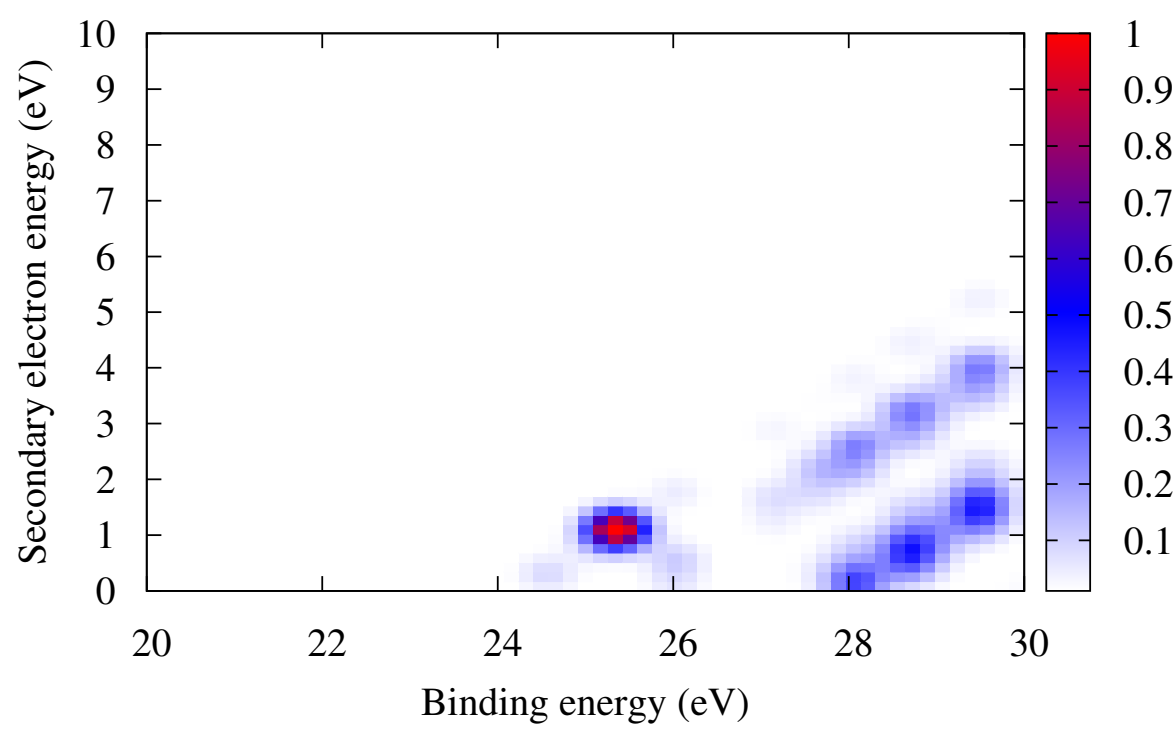

Figure 3: Electron-electron coincidence spectrum of isolated benzene molecule.

geometry of the conformers than the single ionization potentials. Indeed, the lowest peaks of the T-shaped and TT-shaped conformers are red-shifted by $+0.3 \mathrm{eV}$ with respect to that of the PD and sandwich dimers. This energy difference can be rationalized with a simple electrostatic model: assuming the charge on each molecule is evenly distributed between the carbon atoms, the Coulombic repulsion energies are around $2.9 \mathrm{eV}$ for the T-shaped and TTshaped dimers and $3.4 \mathrm{eV}$ for the $\mathrm{PD}$ and sandwich geometries. Another strong difference between the two kinds of conformers is seen in the energy region between 25 and $26 \mathrm{eV}$ : there are no states for the PD and sandwich conformers while the states of the T-shaped and TT-shaped dimers cover this region. However, the DIP of the two T-shaped conformers on one hand and that of the PD and sandwich geometries on the other hand are similar.

The electron-electron coincidence spectra of all geometries are shown in Fig. 5. Compared to the isolated molecule, the spectra exhibit an additional diagonal structure (indicated by a dashed-dotted line) which corresponds to ICD channels. The lower right parts comprise peaks corresponding to both Auger and ICD channels. The spectra show characteristic 
peaks of each species. First, the upper diagonal allows to distinguish between the isolated molecule and the dimers. Furthermore, this line is shifted by $+0.3 \mathrm{eV}$ for the T-shaped and TT-shaped conformers with respect to the PD and sandwich conformers, as expected from the DIP discussed above. Because of this energy shift, a rather intense ICD peak is seen at $B E=20-21 \mathrm{eV}$ and $E=0-1 \mathrm{eV}$ for the T-shaped and TT-shaped conformers while this peak is three times weaker for the PD and even absent for the sandwich conformer. An appearance of a peak in this energy region can thus be unambiguously assigned to the former conformers. Another strong signature of the T-shaped and TT-shaped conformers is seen in the Auger peak at $B E=25-26 \mathrm{eV}$ and $E=0-1 \mathrm{eV}$ (indicated by a red dashed arrow) which is only present for these two geometries. Finally, the two parallel geometries can also be separated since the spectrum for the sandwich geometry exhibits an intense peak at around $B E=22 \mathrm{eV}$ and $E=2 \mathrm{eV}$ (indicated by a black arrow) which is absent in the PD spectrum. The spectra of the T-shaped and TT-shaped dimers are however too similar to distinguish both conformers.

\section{Conclusions}

In conclusion, we have investigated the relaxation pathways after inner-valence ionization of the benzene molecule and the lowest conformers of the benzene dimer. For the molecule, Auger decay opens for states of binding energy above $24.65 \mathrm{eV}$ while for the dimers another decay channel (ICD) opens already at energies above $20 \mathrm{eV}$. Despite the weak interactions between the molecules and the small energy differences between the different conformers, the kinetic energy distributions of the secondary electrons emitted during the electronic decay processes are characteristic of the conformational isomers of benzene dimer. It should be noted that the differences in the coincidence spectra of the conformers can readily be used to experimentally identify the structures since current electron spectrometers and light sources allow to achieve energy resolution below these differences (see for example ${ }^{41,42}$ and 

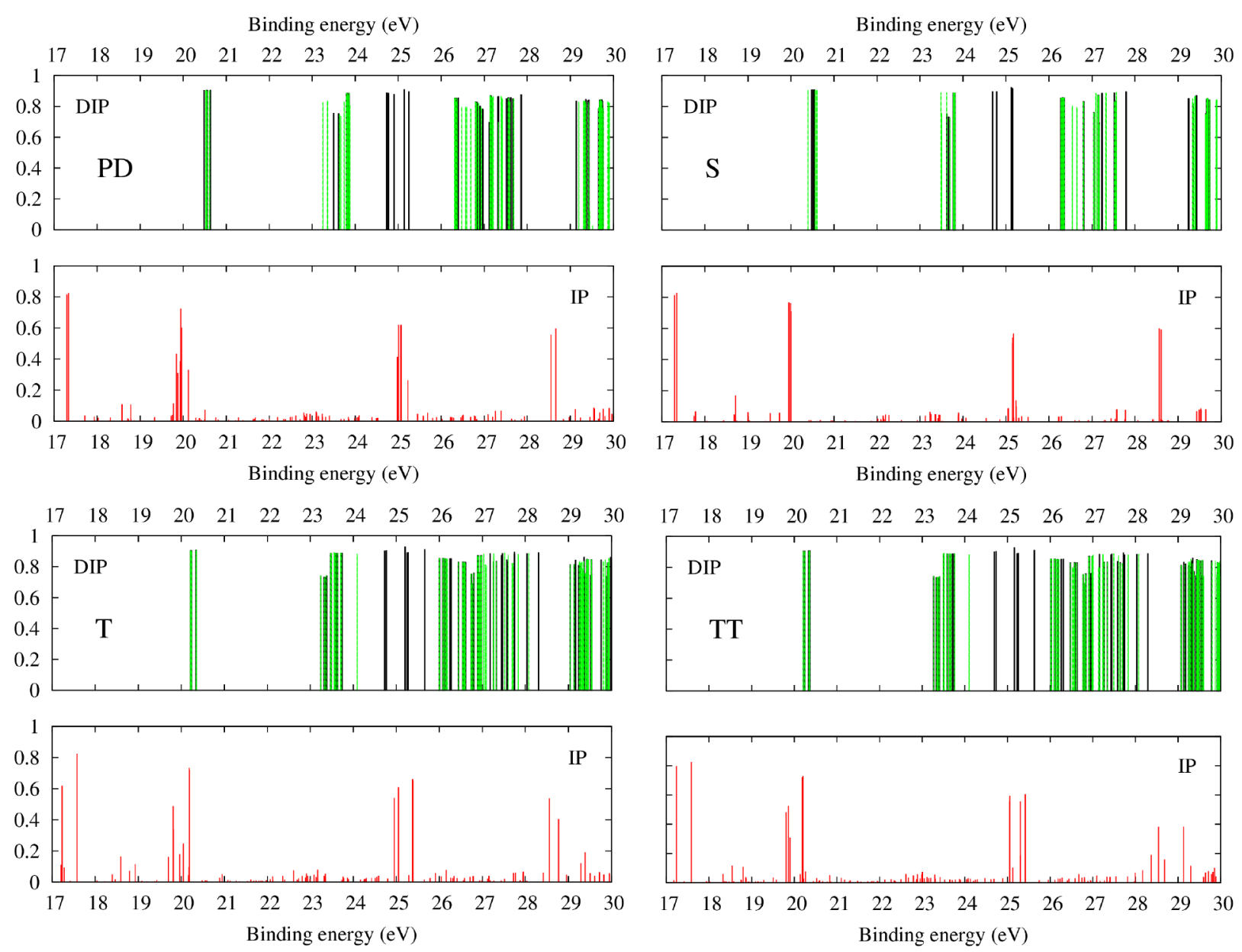

Figure 4: Single and double ionization potentials of the benzene dimer conformers. Singlet and triplet doubly-ionized states are indicated as black and green lines, respectively.

references therein). ICD and Auger decay thus provide a powerful spectroscopic tool for probing weakly-bound molecular clusters.

\section{Acknowledgement}

This project has received funding from the Research Executive Agency (REA) under the European Union's Horizon 2020 research and innovation programme Grant agreement No 705515 and from the LabEx MiChem part of French state funds managed by the ANR within the 'Investissements d'Avenir' program under reference ANR-11-IDEX-0004-02. 

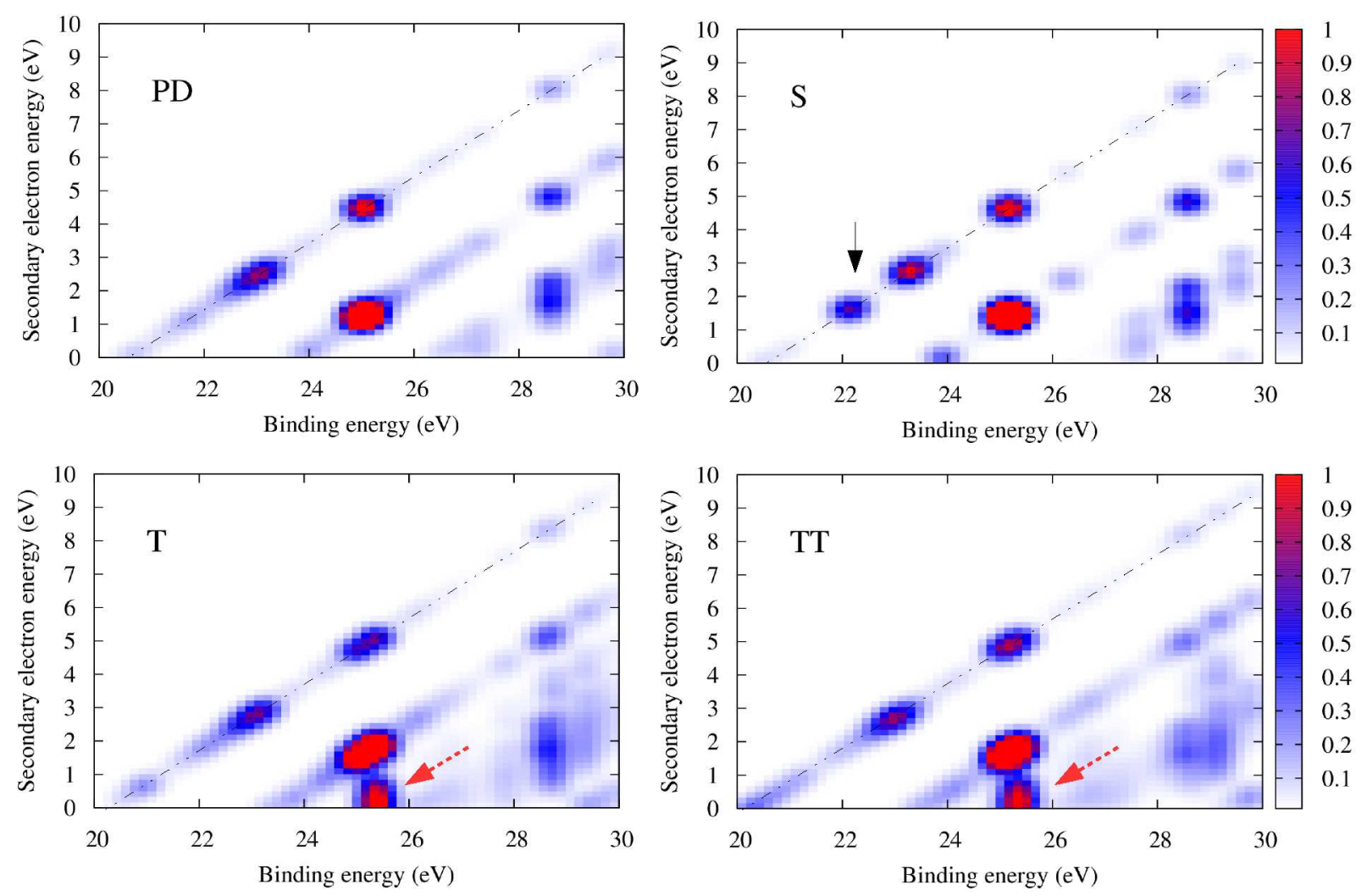

Figure 5: Electron-electron coincidence spectrum of the benzene dimer conformers in the region between 20 and $30 \mathrm{eV}$ corresponding to ionization from inner-valence orbitals.

\section{References}

(1) Hunter, C. A. Meldola Lecture. The Role of Aromatic Interactions in Molecular Recognition. Chem. Soc. Rev. 1994, 23, 101-109.

(2) Burley, S.; Petsko, G. Aromatic-Aromatic Interaction: a Mechanism of Protein Structure Stabilization. Science 1985, 229, 23-28.

(3) Riley, K. E.; Hobza, P. On the Importance and Origin of Aromatic Interactions in Chemistry and Biodisciplines. Acc. Chem. Res 2013, 46, 927-936.

(4) Claessens, C. G.; Stoddart, J. F. $\pi-\pi$ Interactions in Self-Assembly. J. Phys. Org. Chem. 1997, 10, 254-272. 
(5) Gadre, S. R.; Yeole, S. D.; Sahu, N. Quantum Chemical Investigations on Molecular Clusters. Chem. Rev. 2014, 114, 12132-12173.

(6) Sinnokrot, M. O.; Sherrill, C. D. Highly Accurate Coupled Cluster Potential Energy Curves for the Benzene Dimer: Sandwich, T-Shaped, and Parallel-Displaced Configurations. J. Phys. Chem. A 2004, 108, 10200-10207.

(7) Park, Y. C.; Lee, J. S. Accurate ab Initio Binding Energies of the Benzene Dimer. J. Phys. Chem. A 2006, 110, 5091-5095.

(8) Miliordos, E.; Aprà, E.; Xantheas, S. S. Benchmark Theoretical Study of the $\pi-\pi$ Binding Energy in the Benzene Dimer. J. Phys. Chem. A 2014, 118, 7568-7578.

(9) Arunan, E.; Gutowsky, H. S. The Rotational Spectrum, Structure and Dynamics of a Benzene Dimer. J. Chem. Phys. 1993, 98, 4294-4296.

(10) Baumgartel, H.; Brutschy, B.; Ruhl, E. Photoionization of Molecular Aggregates. Phys. Scr. 1990, 1990, 78.

(11) Bradeanu, I. L.; Flesch, R.; Kosugi, N.; Pavlychev, A. A.; Ruhl, E. C 1s $\rightarrow \pi^{*}$ Excitation in Variable Size Benzene Clusters. Phys. Chem. Chem. Phys. 2006, 8, 1906-1913.

(12) Flesch, R.; Serdaroglu, E.; Blobner, F.; Feulner, P.; Brykalova, X. O.; Pavlychev, A. A.; Kosugi, N.; Ruhl, E. Gas-to-solid Shift of C 1s-excited Benzene. Phys. Chem. Chem. Phys. 2012, 14, 9397-9402.

(13) Cederbaum, L. S.; Zobeley, J.; Tarantelli, F. Giant Intermolecular Decay and Fragmentation of Clusters. Phys. Rev. Lett. 1997, 79, 4778-4781.

(14) Marburger, S.; Kugeler, O.; Hergenhahn, U.; Möller, T. Experimental Evidence for Interatomic Coulombic Decay in Ne Clusters. Phys. Rev. Lett. 2003, 90, 203401. 
(15) Jahnke, T.; Czasch, A.; Schöffler, M. S.; Schössler, S.; Knapp, A.; Käsz, M.; Titze, J.; Wimmer, C.; Kreidi, K.; Grisenti, R. E. et al. Experimental Observation of Interatomic Coulombic Decay in Neon Dimers. Phys. Rev. Lett. 2004, 93, 163401.

(16) See http://www.pci.uni-heidelberg.de/tc/usr/icd/ICD.refbase.html for the complete list of ICD papers.

(17) Sisourat, N.; Kryzhevoi, N. V.; Kolorenč, P.; Scheit, S.; Jahnke, T.; Cederbaum, L. S. Ultralong-range Energy Transfer by Interatomic Coulombic Decay in an Extreme Quantum System. Nat. Phys. 2010, 6, 508-511.

(18) Havermeier, T.; Jahnke, T.; Kreidi, K.; Wallauer, R.; Voss, S.; Schöfller, M.; Schössler, S.; Foucar, L.; Neumann, N.; Titze, J. et al. Interatomic Coulombic Decay following Photoionization of the Helium Dimer: Observation of Vibrational Structure. Phys. Rev. Lett. 2010, 104, 133401.

(19) Fasshauer, E.; Förstel, M.; Pallmann, S.; Pernpointner, M.; Hergenhahn, U. Using ICD for Structural Analysis of Clusters: a Case Study on NeAr clusters. New J. Phys. 2014, $16,103026$.

(20) Werner, H.-J.; Knowles, P. J.; Knizia, G.; Manby, F. R.; Schütz, M. Molpro: a Generalpurpose Quantum Chemistry Program Package. WIREs Comput. Mol. Sci. 2012, 2, $242-253$.

(21) Werner, H.-J.; Knowles, P. J.; Knizia, G.; Manby, F. R.; Schütz, M.; Celani, P.; Györffy, W.; Kats, D.; Korona, T.; Lindh, R. et al. MOLPRO, version 2015.1, a package of ab initio programs.

(22) Pitoňák, M.; Neogrády, P.; Řezáč, J.; Jurečka, P.; Urban, M.; Hobza, P. Benzene Dimer: High-Level Wave Function and Density Functional Theory Calculations. J. Chem. Th. Comput. 2008, 4, 1829-1834. 
(23) Danovich, D. Green's Function Methods for Calculating Ionization Potentials, Electron Affinities, and Excitation Energies. WIREs Comput. Mol. Sci. 2011, 1, 377-387.

(24) Schirmer, J.; Trofimov, A. B.; Stelter, G. A Non-Dyson Third-order Approximation Scheme for the Electron Propagator. J. Chem. Phys. 1998, 109, 4734-4744.

(25) Trofimov, A. B.; Schirmer, J. Molecular Ionization Energies and Ground- and Ionicstate Properties using a Non-Dyson Electron Propagator Approach. J. Chem. Phys. 2005, 123, 144115.

(26) Schirmer, J.; Barth, A. Higher-order Approximations for the Particle-Particle Propagator. Z. Phys. A 1984, 317, 267-279.

(27) Tarantelli, F. The Calculation of Molecular Double Ionization Spectra by Green's Functions. Chem. Phys. 2006, 329, 11-21, Electron Correlation and Multimode Dynamics in Molecules (in honour of Lorenz S. Cederbaum).

(28) Velkov, Y.; Miteva, T.; Sisourat, N.; Schirmer, J. Intermediate State Representation Approach to Physical Properties of Dicationic States. J. Chem. Phys. 2011, 135, 154113.

(29) Krishnan, R.; Binkley, J. S.; Seeger, R.; Pople, J. A. Self-consistent Molecular Orbital Methods. XX. A Basis Set for Correlated Wave Functions. J. Chem. Phys. 1980, 72, 650-654.

(30) Schmidt, M. W.; Baldridge, K. K.; Boatz, J. A.; Elbert, S. T.; Gordon, M. S.; Jensen, J. H.; Koseki, S.; Matsunaga, N.; Nguyen, K. A.; Su, S. et al. General Atomic and Molecular Electronic Structure System. J. Comput. Chem. 1993, 14, 1347-1363.

(31) Zobeley, J.; Santra, R.; Cederbaum, L. S. Electronic Decay in Weakly Bound Heteroclusters: Energy Transfer versus Electron Transfer. J. Chem. Phys. 2001, 115, 50765088. 
(32) Sakai, K.; Stoychev, S.; Ouchi, T.; Higuchi, I.; Schöffler, M.; Mazza, T.; Fukuzawa, H.; Nagaya, K.; Yao, M.; Tamenori, Y. et al. Electron-Transfer-Mediated Decay and Interatomic Coulombic Decay from the Triply Ionized States in Argon Dimers. Phys. Rev. Lett. 2011, 106, 033401.

(33) Santra, R.; Cederbaum, L. S.; Meyer, H.-D. Electronic Decay of Molecular Clusters: Non-Stationary States Computed by Standard Quantum Chemistry Methods. Chem. Phys. Lett. 1999, 303, 413-419.

(34) Agren, H. On the Interpretation of Molecular Valence Auger Spectra. J. Chem. Phys. 1981, 75, 1267-1283.

(35) Tarantelli, F.; Sgamellotti, A.; Cederbaum, L. S.; Schirmer, J. Theoretical Investigation of Many Dicationic States and the Auger Spectrum of Benzene. J. Chem. Phys. 1987, $86,2201-2206$.

(36) Averbukh, V.; Müller, I. B.; Cederbaum, L. S. Mechanism of Interatomic Coulombic Decay in Clusters. Phys. Rev. Lett. 2004, 93, 263002.

(37) Baltzer, P.; Karlsson, L.; Wannberg, B.; Ohrwall, G.; Holland, D.; MacDonald, M.; Hayes, M.; von Niessen, W. An Experimental and Theoretical Study of the Valence Shell Photoelectron Spectrum of the Benzene Molecule. Chem. Phys. 1997, 224, 95119.

(38) Yencha, A. J.; Hall, R. I.; Avaldi, L.; Dawber, G.; McConkey, A. G.; MacDonald, M. A.; King, G. C. Threshold Photoelectron Spectroscopy of Benzene up to $26.5 \mathrm{eV}$. Canad. J. Chem. 2004, 82, 1061-1066.

(39) Eland, J. H. Spectra of the Dications of Benzene, Naphthalene and Azulene. Chem. Phys. 2008, 345, 82-86. 
(40) Cederbaum, L. S.; Domcke, W.; Schirmer, J.; Niessen, W. V. Correlation Effects in the Ionization of Molecules: Breakdown of the Molecular Orbital Picture. Adv. Chem. Phys. 1986, 65, 115-159.

(41) Penent, F.; Palaudoux, J.; Lablanquie, P.; Andric, L.; Feifel, R.; Eland, J. H. D. Multielectron Spectroscopy: The Xenon 4d Hole Double Auger Decay. Phys. Rev. Lett. 2005, 95,083002 .

(42) Ulrich, V.; Barth, S.; Lischke, T.; Joshi, S.; Arion, T.; Mucke, M.; Förstel, M.; Bradshaw, A. M.; Hergenhahn, U. Photoelectron-Auger Electron Coincidence Spectroscopy of Free Molecules: New Experiments. J. Elec. Spect. Rel. Phen. 2011, 183, 70-79

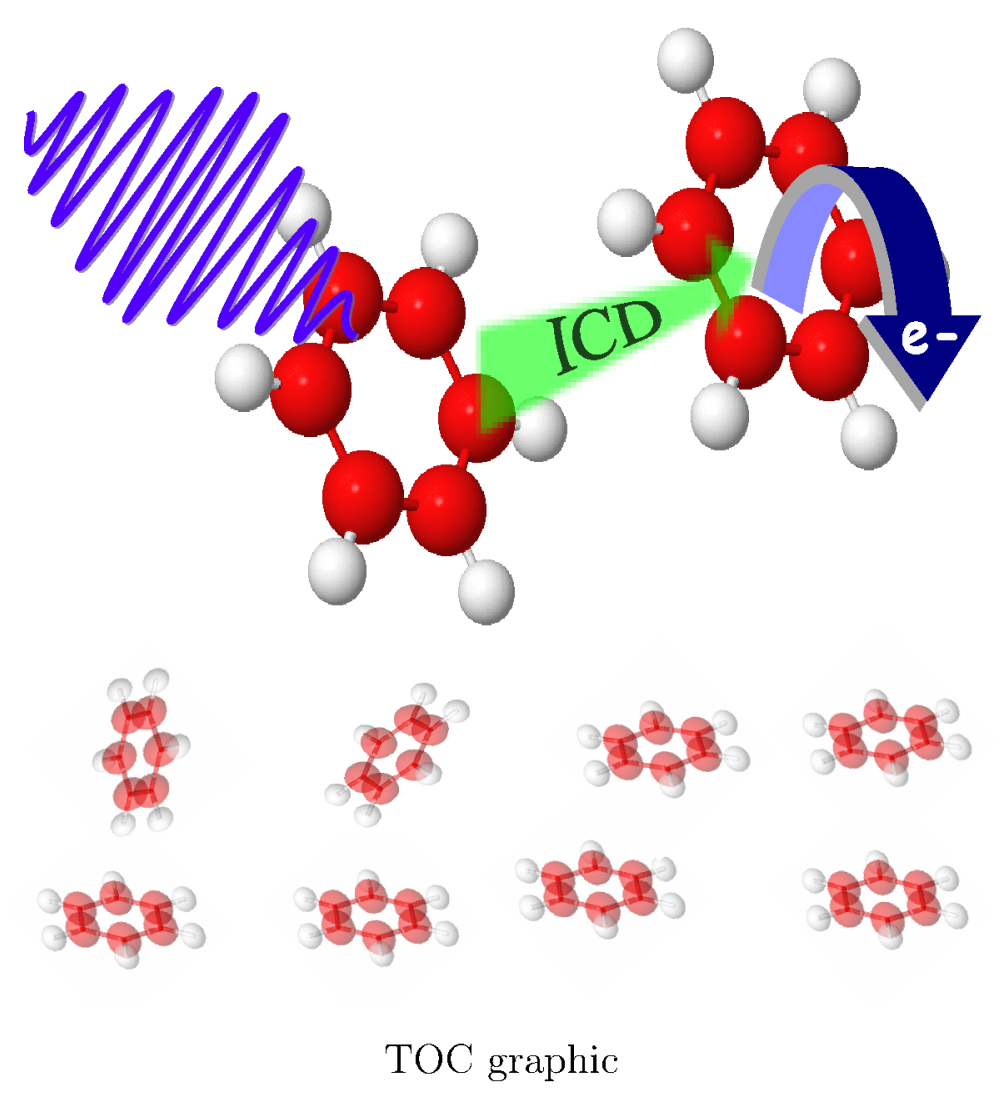




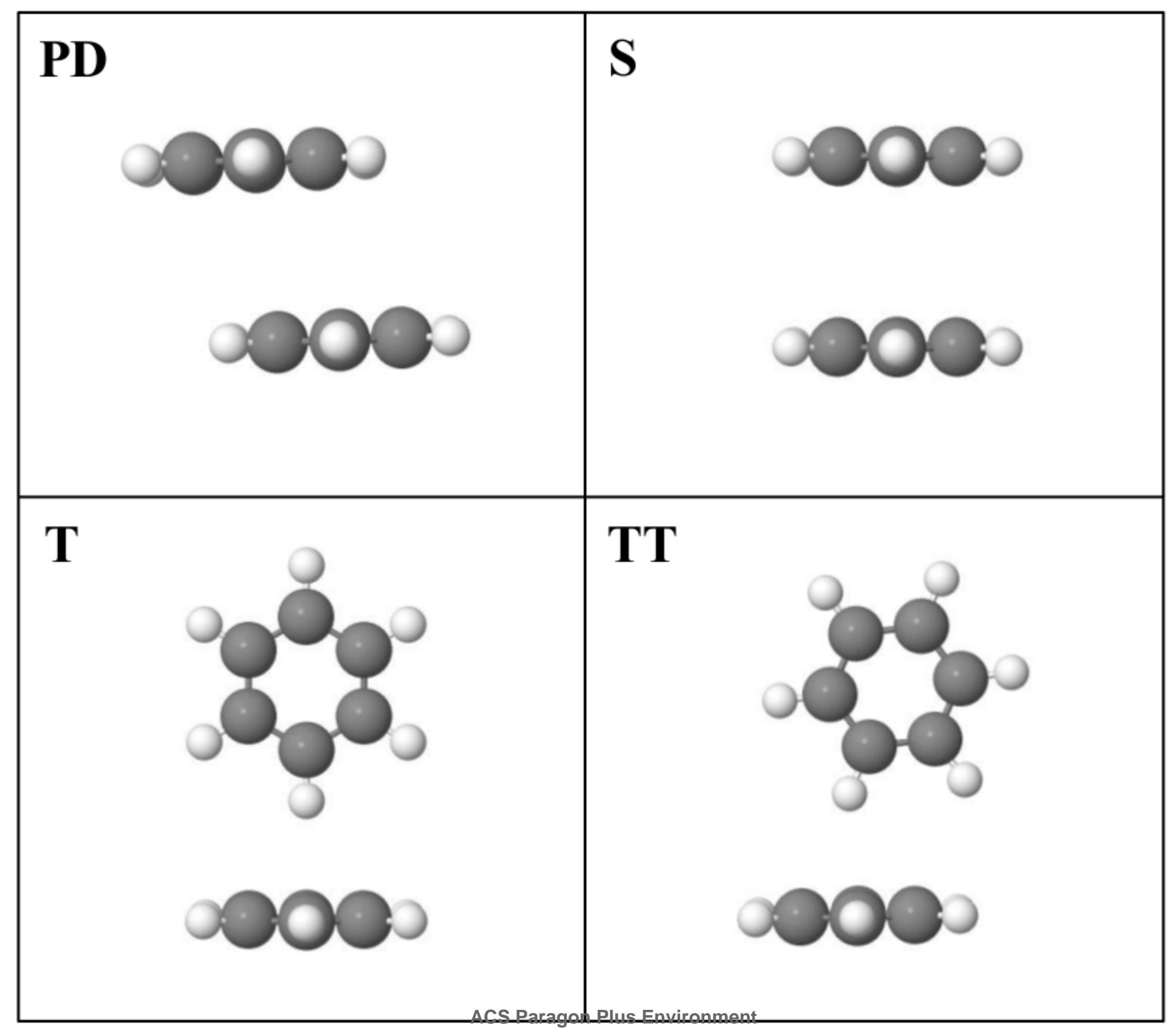


Binding energy $(\mathrm{eV})$

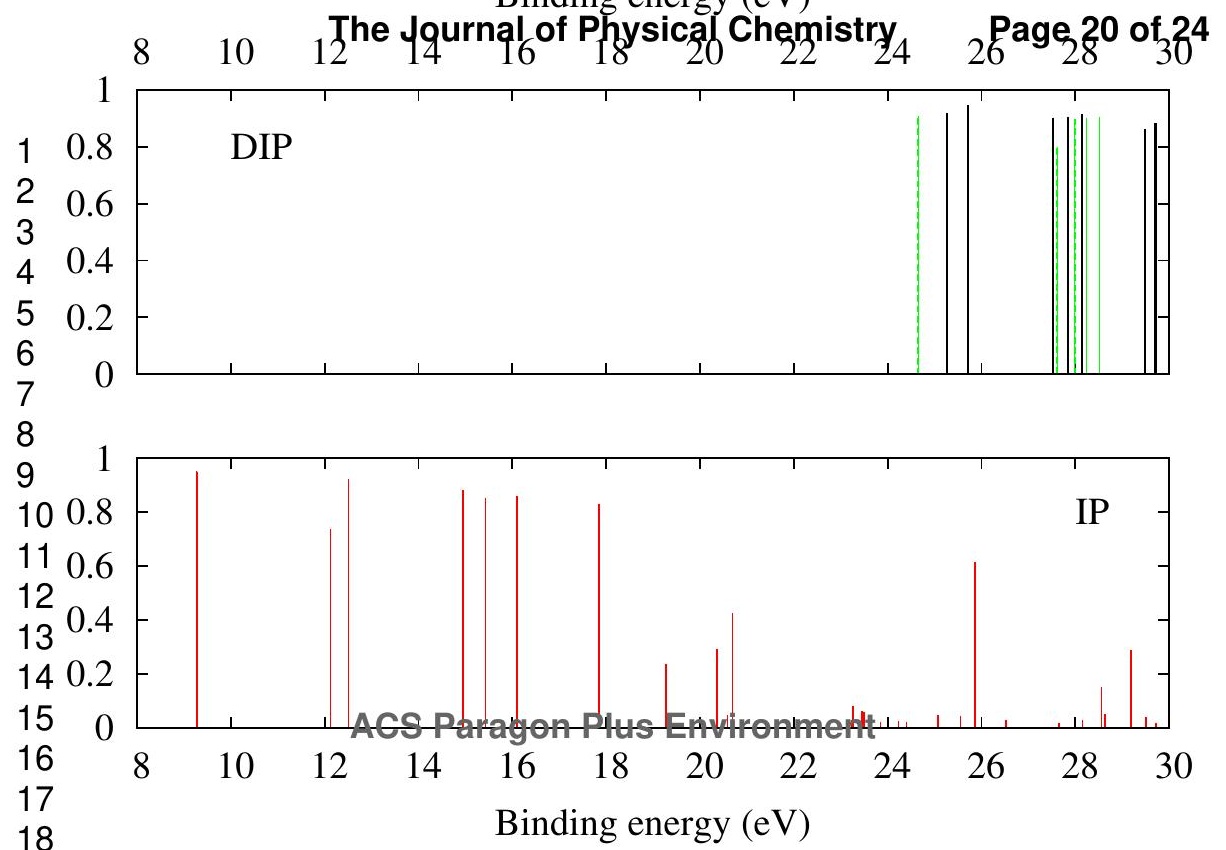




\section{Page 21 of 24 The Journal of Physical Chemistry}

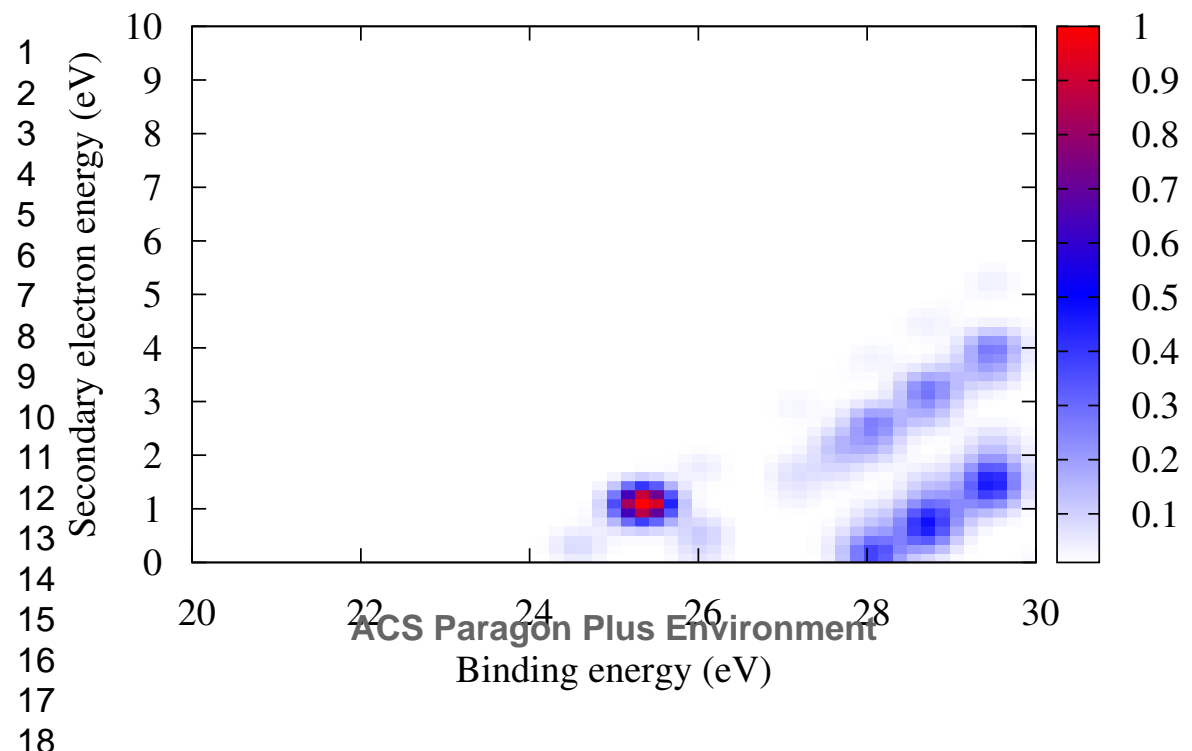




\section{Page 23 of 24}

The Journal of Physical Chemistry
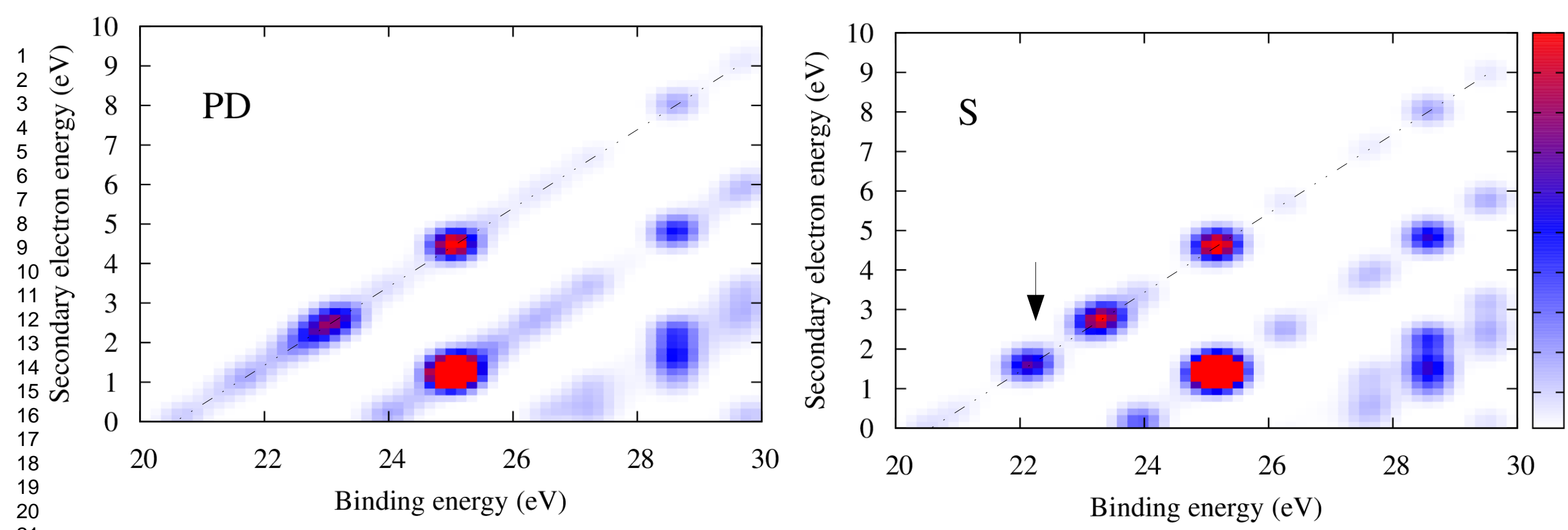

1
0.9
0.8
0.7
0.6
0.5
0.4
0.3
0.2
0.1
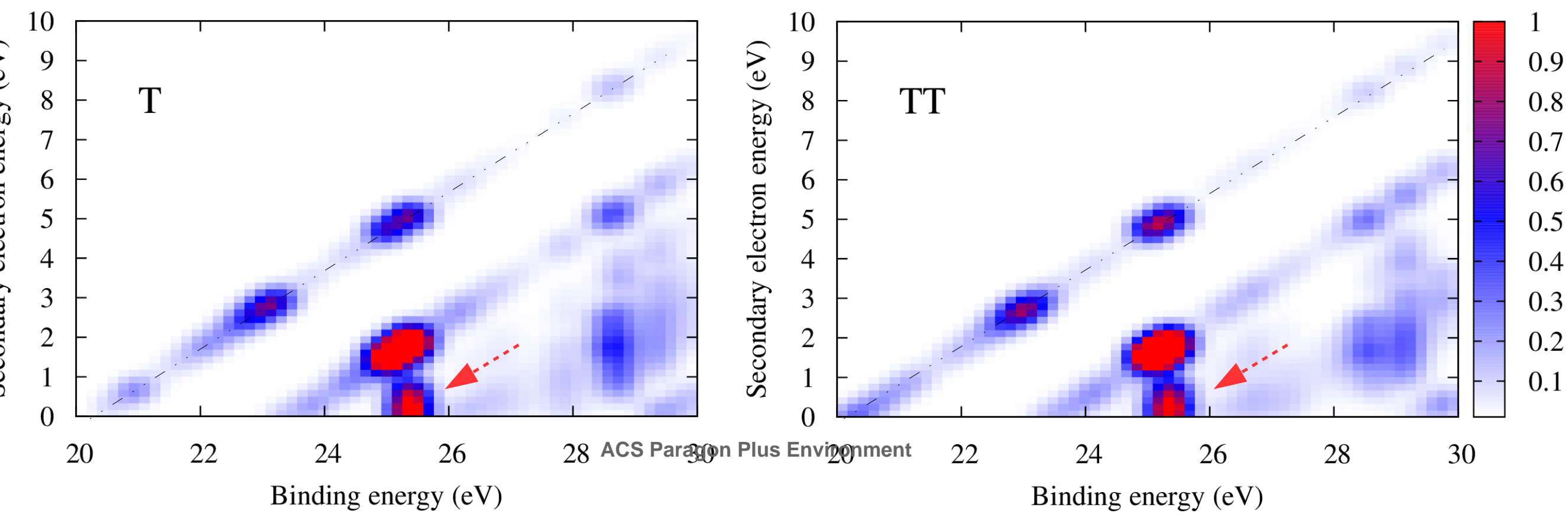
